\title{
Las infancias transnacionales desde las perspectivas postcolonial y decolonial 1
}

Transnational childhoods from postcolonial and decolonial perspectives

\author{
Manfred Liebel \\ Universidad de Ciencias Aplicadas de Postdam \\ mliebel@ina-fu.org
}

\section{Resumen}

En este artículo se discute que la existencia de los niños, así como las ideas y conceptos de la infancia que han surgido en Europa desde finales de la Edad Media, están estrechamente ligados a los procesos de colonización de otros continentes. Se identifican tres dimensiones de la transnacionalidad desde las perspectivas postcolonial y decolonial: interdependencia del proyecto de la infancia burguesa y la colonización de los continentes extranjeros; la destrucción y desvalorización de las infancias en las antiguas regiones coloniales; y, la problematización del proyecto de la infancia eurocéntrico-imperial. El artículo cuestiona que el modelo europeo de infancia sea visto como la forma más elevada de civilización y que se utilice como un criterio global y transnacional para evaluar otras formas de infancias alrededor del mundo, puesto que dicho modelo se impuso a través de un proceso (post)colonial-imperial.

Palabras Clave: Infancia, Transnacionalismo, Colonialización, Eurocentrismo, Estudios Post-coloniales, Descolonización

\begin{abstract}
This paper discusses the thesis that the existence of children, as well as the ideas and concepts of childhood that have emerged in Europe since the late Middle Age, are closely linked to the colonization of other parts of the world. Three dimensions of transnationality are identified from postcolonial and decolonial perspectives: Interdependence of the bourgeois childhood project and the colonization of foreign continents; destruction and devaluation of childhoods in the former colonial regions; problematization of the eurocentric-imperial childhood project. The assumption that the childhood pattern created in Europe represents the highest form of civilization and can serve as a global, transnational yardstick is rejected as (post)colonial-imperial presumption.
\end{abstract}

Keywords: Childhood, Transnationalism, Colonialization, Eurocentrism, Postcolonial Studies, Decolonization

Manfred Liebel

Las infancias transnacionales desde las perspectivas postcolonial y decolonial. Autoctonía. Revista de Ciencias Sociales e Historia, Vol. III, N², Julio-Diciembre 2019, 97-110 ISSN 0719-8213

DOI: http://doi.org/10.23854/autoc.v3i2.134 


\section{Introducción}

Las cuestiones de transnacionalidad o transnacionalismo se han abordado principalmente en la investigación sobre las migraciones durante los últimos dos decenios ("transnational turn"). En términos generales, el transnacionalismo se entiende como los "fenómenos transfronterizos que, anclados localmente en diferentes sociedades nacionales, constituyen relaciones sociales, redes sociales o espacios sociales relativamente permanentes y densos" y "tienen una nueva cualidad histórica debido a su frecuencia y densidad" (Pries, 2010: 13). La investigación sobre las infancias transnacionales, que se encuentra en la intersección de la investigación sobre la migración y la infancia, comenzó hace sólo unos pocos años y sigue siendo relativamente escasa (Rohr, 2013; Pavez-Soto, 2013; Emond y Esser, 2015).

Siguiendo esa línea de análisis, aquí se discutirá este tema en forma de un ensayo teórico reflexivo desde la perspectiva de la historia de la colonización, los enfoques postcoloniales, decoloniales y las construcciones sociales de la infancia (Liebel, 2019; Schibotto, 2015). Para ello, se parte de la siguiente tesis central (que será desagregada en ciertos ejes temáticos) y que está inspirada en los estudios postcoloniales y decoloniales: Los niños, en tanto sujetos, así como las ideas y los conceptos de la infancia que han surgido en Europa desde finales de la Edad Media, están estrechamente ligados a la colonización de otras partes del mundo. La afirmación común de que sólo hay una infancia que representa, por así decirlo, la forma más elevada de civilización y que puede servir como un estándar mundial y transnacional, es en sí misma una presunción (post)colonial-imperial.

En la historia moderna de las infancias, se pueden identificar a grandes rasgos, tres ejes temáticos sobre los cuales ha versado el tema de transnacionalidad o el transnacionalismo desde una perspectiva postcolonial, a saber: a) la interdependencia del proyecto de la infancia "moderna" surgiendo con las sociedades capitalistas-burguesas en Europa y la colonización de los continentes extranjeros; b) la destrucción y desvalorización de las infancias en las antiguas regiones coloniales; y c) la problematización del proyecto de la infancia eurocéntrico-imperial. A continuación pasamos a revisar cada uno de estos ejes temáticos que sustentan nuestra tesis central de discusión.

\section{Infancia moderna y colonización}

En este eje temático se reflexionará a partir de la primera tesis: El modelo de infancia que surgió en el marco de la sociedad burguesa y el estado de desarrollo nacional en Europa es un proyecto colonial $y$, al mismo tiempo, imperial. La colonización se basa en la 
explotación económica intensiva de los territorios colonizados que se consideraban, aparentemente, vacíos e incivilizados (terra nullius o tabula rasa). Estas lógicas de colonización de territorios también han influido en las formas de concebir a la infancia moderna, entedida como un territorio vacío e incivilizado.

Una característica esencial del proyecto moderno de la infancia europea es imaginar a la infancia como una etapa preliminar imperfecta y evolutiva, respecto de la edad adulta, la que se considera perfecta y desarrollada. Es interesante notar que el concepto de infancia separada del mundo adulto, "libre" de tareas productivas implica que también es marginada de la sociedad (adulta). No olvidemos que este concepto de infancia surgió paralelamente al "descubrimiento" y a la colonización del mundo fuera de Europa (desde finales del siglo XV). Por un lado, el sometimiento y la explotación de las colonias -primero en América y, posteriormente, en África y Asia-constituyeron la condición material para que surgiera dicho concepto, pues dieron origen a que en la "madre patria" naciese una clase social que gozaba de bienestar material y, por lo tanto, podía permitirse privatizar a sus niños, entregándoles un servicio privado de protección y cuidado.

Por otro lado, se podría pensar que el sometimiento de las personas de las colonias sirvió de modelo para educar a la niñez local bajo esa misma lógica de dominación, independiente de si pertenecía a la clase dominante o a los grupos sociales subalternos. De tal manera, podríamos hablar de una colonización de la infancia o de la infancia moderna como una especie de sujeto colonizado. Esa visión sirvió de modelo también a las tempranas ciencias que comenzaron a especializarse en la infancia, las cuales apuntaban al control y a la perfección de la infancia. A la inversa, la construcción de la infancia como etapa previa, inmadura y menor a la adultez sirvió como una matriz para sustentar prácticas de degradación de seres humanos de las colonias, que, si bien, no eran niñas y niños, sino de otras edades, se les concebía como seres inmaduros, necesitados de desarrollo y que permanecían en un estado de infancia. Por eso, se recurrió a la metáfora de ver a los "pueblos primitivos" como niños, a fin de encontrar una expresión adecuada, entendiendo que esas personas se encontraban en una etapa de desarrollo inferior, porque eran percibidos como "salvajes" e "incivilizados", tal como eran vistos los niños y las niñas. Este concepto se refleja por ejemplo en la famosa sentencia de Hegel que calificó a África como un "país infantil“ (Hegel, [1802]1989).

Las interrelaciones entre el nuevo concepto de la infancia y la colonización de los pueblos y continentes extranjeros ya se habían establecido previamente en las ideas de filósofos liberales, tales como el inglés John Locke (1632-1704) o el francés Jean-Jacques Rousseau (1712-1778), cuyas ideas han sido fundamentales para entender la historia de la infancia moderna. En su Ensayo sobre el entendimiento humano (1690), Locke describió al niño como "tabla rasa", o en términos 
más populares, "papel en blanco" 2 , atribuyendo a los padres y a los maestros una gran responsabilidad por lo que se inscribiría en ese papel en blanco. Al mismo tiempo, este concepto era de suma importancia para el emprendimiento imperial, puesto que la idea de ver a los pueblos colonizados como un espacio vacío funcionó, finalmente, como un prerrequisito para la colonización, entendida como civilización ${ }^{3}$. Mientras Locke imaginaba que un niño recién nacido era un espacio vacío que había que llenar, cien años más tarde, Rousseau describió al niño como "naturaleza pura", que en sí ya constituye un valor y que es corrompido por la civilización, en su obra Emilio, o De la educación ([1762]1998). Entonces, en Rousseau, el paralelismo entre la infancia y la colonización está condensado en la idea del "buen salvaje". En efecto, ambos conceptos justifican el actuar colonial en un sentido paternalista porque tanto la inocencia de la naturaleza como también la tabla vacía del niño no formado equivalen a la ausencia de significado y a la exclusión social. Según Uday Singh Mehta (1999: 48), las estrategias excluyentes implican un "infantilismo civilizacional". Ni el niño ni el sujeto colonial tienen acceso a significados y a pertenecer a la categoría de sujeto (adulto, independiente y racional), eso solo sucede mediante el proceso de colonización, civilización y educación.

En este sentido, el colonialismo siempre ha sido un "colonialismo educativo" (Osterhammel, 2005: 110) que se atribuía a sí mismo la tarea de liberar a los colonizados de la tiranía de la naturaleza y de la oscuridad intelectual. Y más aún, la equiparación de los colonizados con el estatus de los niños permitió edulcorar esta pretensión como una obligación moral o, como se le ha denominado, la "carga del hombre blanco" (Kipling, 1899; Walsh, 2010)4. De hecho, la colonización se ha entendido como un proceso civilizatorio con tintes caritativos, por ejemplo, en frases como estas: "El dominio colonial fue glorificado como regalo y acto de misericordia de la civilización, como una especie de intervención humanitaria permanente" (Osterhammel, 2005: 115). Para ello, uno de los instrumentos más importantes fueron las escuelas que estaban dirigidas por el Estado o por misioneros y, en algunos casos, por instituciones privadas no misioneras. Las escuelas apuntaban -y todavía apuntan- a transmitir un determinado tipo de pensamiento y de moral que va mucho más allá de solamente aprender a leer y a escribir y que podríamos calificar como una especie de "tecnología moral" (Wells, 2015: 108), "violencia epistémica” (Cannella y Viruru, 2004: 2; De Sousa Santos, 2009) o "colonización de conciencia" (Comaroff y Comaroff, 2008).

La instrumentalización del concepto eurocéntrico de infancia moderna, entendido como legitimación y justificación de la conquista colonial, tiene una notable correspondencia en la visión de la infancia como colonia u sujeto/objeto colonizado. De hecho, desde los años 1960, el uso del término colonización ya no se limita a áreas geográficas generalmente extraeuropeas y a sus respectivas poblaciones, sino, también a la estructura interna de las sociedades y de las per- 
sonas que las conforman. Así, a principios de los años 1980, el filósofo Jürgen Habermas (1981) planteó el concepto de la "colonización interna" de los mundos de vida. A partir de la suposición que en el capitalismo tardío los subsistemas fundamentales de economía y Estado se desprenden de los mundos de vida, la propia vida de los miembros de una sociedad es colonizada, de la misma manera que los colonizadores penetran a una sociedad tribal para apoderarse de su vida. Con ello, Habermas identifica que se ponen en peligro las funciones socializadoras y generadoras de identidad de los mundos de vida.

Algunos documentos de esa época comprenden la opresión de la infancia como una forma de colonización, estableciendo una relación con el colonialismo. El antropólogo y psicoanalista suizo Gérard Mendel publicó -primero en francés (Mendel, 1971) y luego traducido al español (Mendel, 1974)- un alegato a favor de la descolonización del niño. Por otro lado, un ensayo del pedagogo austriaco Peter Gstettner se titula: La conquista del niño por la ciencia. De la historia de la disciplina (Gstettner, 1981), donde el autor hace referencia explícita a la historia del colonialismo y utiliza el ejemplo de las nuevas ciencias pedagógicas y psicológicas de la infancia para ilustrar la relación de la etnología al servicio de la colonización. La investigación de este autor se basa en la tesis de que "la conquista científica de territorios desconocidos precede a la conquista del alma del niño" (Gstettner, 1981: 15). Esto lo demuestra especialmente en la historia de la psicología evolutiva, pero también en la conceptualización de la infancia que podemos encontrar en todas las ciencias.

La construcción de una infancia estrictamente distinguida y separada del mundo adulto está necesariamente asociada a la ambivalencia. Incluso si se sigue con la intención de dotar al niño de "sus propios espacios" y de aliviarlo temporalmente de la "seriedad de la vida" o de concederle una protección especial, inevitablemente se devalúan sus competencias y su estatus social. En estas circunstancias, el "privilegio" de ser salvado y protegido se produce a expensas de la independencia y el reconocimiento de la especificidad o la diferencia se convierte en desigualdad. Así lo demuestra el hecho de que los niños a veces se alegran de no verse abrumados por las obligaciones, pero, tarde o temprano, sienten que ser niño es una forma de desprecio y ya no quieren ser considerados como "niños".

\section{Infancias en las antiguas regiones coloniales}

En este apartado temático se reflexionará sobre la segunda tesis: En la globalización postcolonial de carácter capitalista, las infancias están siendo remodeladas y unificadas, lo que se expresa en una creciente desigualdad, al tiempo que surge una conquista postcolonial de las infancias del Sur Glo$\mathrm{bal}^{5}$, en un escenario de hegemonía de un modo de vida imperial. Las infancias del Sur Global hoy en día están siendo destruidas y devaluadas ideológicamente, fruto de la creciente desigualdad social. La desigualdad, la destrucción y la devaluación aumentan la movilidad y la migración; por lo tan- 
to, se crean nuevos patrones globales e híbridos de infancia.

La compresión espacial del mundo a través de los procesos económicos y tecnológicos, así como las normas jurídicas internacionales, sin duda influyen en las ideas de qué es una "buena infancia" y eso incide en la vida concreta de los niños; podríamos decir que la actual globalización los acerca más y, quizás, incluso, los hace más parecidos. Una pregunta pertinente sería, por tanto: ¿cómo podemos nombrar a las infancias que surgen en la globalización? ¿Sería justificable describir a las niñas y los niños de hoy en día como más occidentalizados, modernizados, secularizados, legalizados, educados o consumidores?

En su intento de concebir una historia global de la infancia, el historiador Peter Stearns (2005; 2006) habla de cómo la infancia fuera de Europa no se ha occidentalizado, sino que ha cambiado de acuerdo a los patrones "del modelo occidental". Por supuesto, no se puede negar que como resultado de la colonización y los modelos occidentales dominantes las escuelas, por ejemplo, están ocupando cada vez más espacios en la vida de los niños y, a su vez, están adquiriendo más importancia para su vida futura. Pero, al igual que ocurre en otros aspectos de la globalización de la infancia, hay que tener en cuenta que esto no se aplica de la misma manera para todos los niños o no siempre adquiere el mismo significado. El aprendizaje escolar, así como la legalización de las relaciones sociales o el uso de las tecnologías digitales, pueden concebirse y practicarse de formas muy diferentes.

Quizás el mayor desafío de la investigación sobre la infancia en la globalización es comprender las conexiones y contradicciones entre las dimensiones globales y locales de la infancia y los modos de vida de los niños, tanto a nivel objetivo (material), como a nivel subjetivo (el pensamiento, los sentimientos y la acción). Los niños están influenciados por lo que está sucediendo en otras partes del mundo, al igual que los jóvenes y los adultos (aunque no de la misma manera), porque hoy en día no hay nichos aislados. Pero la forma en que la globalización influye las vidas de cual dependerá de las condiciones de vida y en qué parte del mundo se viva, -esto no debe pasarse por alto-. La globalización de las infancias no es un proceso unilineal ni absolutamente inevitable, sino que implica muchas interdependencias, no produce una sola "infancia global" homogénea, sino muchas y muy diferentes "infancias globales" (Cregan y Cuthbert, 2014). Estas infancias globales están influenciadas de una manera particular por los diferentes patrones de colonialización practicados por las potencias coloniales hispanas, británicas y francesas, por un lado, y por las culturas precoloniales de los pueblos colonizados y sus reacciones a la dominación colonial, por el otro.

No debe pasarse por alto que las interdependencias en la formación de las infancias globalizadas están arraigadas a una estructura de poder mundial extremadamente desigual. Lo desigual 
que pueden ser estas interdependencias globales es más evidente en la brecha cada vez mayor entre las regiones ricas y pobres del mundo, debido a las menores oportunidades de vida que tienen los niños que viven en las partes más pobres del mundo. Sin embargo, esto también se aplica a las condiciones dentro de los propios países y las regiones del Sur Global, que también se caracterizan por una considerable desigualdad social. La creciente desigualdad en todo el mundo refleja la distribución desigual del poder entre el Norte y el Sur, que surgió justamente durante la época colonial y continúa hasta el día de hoy, de forma institucional. Ya no se expresa abiertamente en la expansión, conquista y dominación colonial, sino que se puede observar en la dependencia menos visible de los Estados nacionales aparentemente independientes en el Sur Global, que a su vez, reproducen internamente la desigualdad social y política.

En la globalización postcolonial y sus efectos en la niñez del Sur Global se deben distinguir los aspectos materiales e ideológicos. En cuanto al aspecto material, por ejemplo, un Informe sobre Desarrollo Humano de las Naciones Unidas (PNUD, 1999: 3) muestra que la desigualdad de ingresos entre la primera quinta parte de la población mundial que vive en los países más ricos y la última quinta parte que vive en los países más pobres ha aumentado más de veinte veces en los últimos 200 años. Otros estudios sobre la distribución mundial de la renta y la riqueza (Milanović, 2012; Piketty, 2014) también muestran que las desigualdades materiales en el con- texto mundial han sido durante mucho tiempo extremadamente altas y es probable que sigan aumentando, a menos que se contrarresten políticamente. La discrepancia entre ricos y pobres nunca ha sido tan grande en la historia de la humanidad.

Stephan Lessenich (2017) resume estos procesos con el término "sociedad de externalización". En consecuencia, las sociedades del Norte siguen ganando prosperidad al tener que apretarse el cinturón y renunciar a una explotación postcolonial típica. Ulrich Brand y Markus Wissen (2017) culpan de ello al "modo de vida imperial". Según ellos, en muchas partes del mundo se

“agravan los fenómenos de crisis como el cambio climático, la destrucción de ecosistemas, la polarización social, el empobrecimiento de muchas personas, la destrucción de las economías locales o las tensiones geopolíticas que hasta hace unos años se suponía que se habían superado con el fin de la Guerra Fría. Además, uno de los principales contribuyentes a estos fenómenos de crisis. [...] Es la forma de vida imperial, que se basa en la exclusividad, porque sólo puede sobrevivir mientras tenga un exterior al que pueda trasladar sus costes. Pero este exterior está disminuyendo, porque cada vez más economías tienen acceso a él y cada vez menos personas están dispuestas o son capaces de soportar los costes de los procesos de externalización" (Brand y Wissen, 2017: 13 y 15). 
La desigual estructura de poder global también se refleja en el hecho de que las formas de vida de los niños y las infancias del Sur Global son devaluadas, ignoradas e invisibilizadas. Los niños son a menudo declarados niños que existen "fuera de la infancia" (Ennew, 2002) o son "niños sin infancia" (UNICEF, 2006) y, en el mejor de los casos, son percibidos con compasión.

Otra consecuencia de esta estructura de poder es que si bien la difusión de las escuelas y los nuevos medios digitales ha aumentado las posibilidades de adquirir información y conocimientos, al mismo tiempo ha devaluado y destruido los conocimientos prácticos que los niños de las culturas originarias, como por ejemplo, las culturas precolombinas, habían adquirido gracias a su proximidad a la naturaleza y a su participación en actividades comunitarias y familiares. Este conocimiento se refería, por ejemplo, a las características de las plantas y los animales y a la necesidad de su cuidado y preservación o al manejo de los riesgos y peligros en el entorno de los niños. Era un conocimiento que resultaba de la experiencia práctica y de su participación en tareas vitales. Hoy en día, estas conexiones están prácticamente rotas, y además han marginado a los niños de las regiones rurales y los han expuesto a la discriminación, como lo han demostrado Cindi Katz $(2004 ; 2012)$ y Sarada Balogapalan (2014) con los ejemplos de Sudán e India, respectivamente. Sin embargo, los niños y los jóvenes siguen siendo importantes para sus comunidades; con sus recién adquiridos y mejores conocimientos, a menudo se transforman en ac- tores que señalan nuevos caminos, actualizando los conocimientos tradicionales y las formas de vida comunitarias.

Otro ejemplo podría ser que la destrucción de los modos de producción tradicionales se ha asociado al robo de tierras, lo que provoca dificultades materiales y a menudo conflictos internos en el Sur Global. Eso tiene como resultado las expulsiones y las migraciones, que pueden ser dentro de los países, como, por ejemplo, desde el campo hacia las ciudades, o hacia los países vecinos y hacia las regiones más ricas del mundo. Los movimientos migratorios provocados por las dificultades materiales y la desigualdad social dan lugar a nuevas formas de infancia, ya sea que los propios niños se conviertan en actores de la migración ("children on the move"), o regresen a sus hogares y sigan dependiendo de sí mismos, de sus parientes o de sus vecinos. Las niñas y los niños que participan en procesos migratorios globales están asociados con riesgos y sufrimientos que ponen en peligro su vida, pero también crean nuevas identidades, nuevas experiencias y nuevos conocimientos. Algunas comunidades y familias del hemisferio sur dependen de los ingresos y del capital cultural y social recientemente adquirido de sus jóvenes migrantes para su supervivencia y progreso. Estamos ante el surgimiento de un fenómeno social sobre las infancias y juventudes transnacionales que nunca antes habíamos visto, al menos, como lo conocemos hoy.

La comprensión eurocéntrica de la infancia no 
hace justicia a estos cambios y los oscurece, en lugar de ayudar a comprenderlos. En el plano normativo, las percepciones idealizadas sobre las condiciones de vida de las niñas y los niños que viven en el Sur Global podría llevar a distorsiones sobre su realidad. Por eso, apelamos a una articulación e integración de conceptos, a través de infancias híbridas o múltiples porque las infancias actuales del Sur Global no son infancias "liberadas" y "separadas", en el sentido del patrón idealizado de la infancia eurocéntrica, sino, que se podría hablar de infancias que están estrechamente relacionadas con el desarrollo de su sociedad y sus desafíos existenciales. Y esto no se limita al Sur Global, sino que se extiende a través de los procesos de migración, la precarización de un número creciente de personas y la disolución de la estricta separación de la esfera del trabajo y la reproducción también en el Norte Global.

\section{Proyecto eurocéntrico-imperial de la infancia}

En este último eje temático vamos a reflexionar sobre la tercera tesis: Las infancias híbridas y globalizadas resultantes de la globalización postcolonial conducen a una problematización del proyecto de infancia eurocéntrica-imperial, la que era vista como una supuesta cúspide del progreso de civilización en el propio Norte Global. La exclusión constitutiva de los niños de la vida adulta y la definición de la infancia como la etapa preparatoria para la vida adulta son cuestionadas fundamentalmente.

El proyecto de infancia y las relaciones genera- cionales que ha dominado en el Norte Global, bajo las repercusiones de la globalización postcolonial, ha estado en tela de juicio desde hace cuatro décadas, aproximadamente. Indicios de ello son, por ejemplo, la preocupación por la "desaparición de la infancia" (Postman, 1988) o, visto desde otra perspectiva, la disminución de la voluntad de los niños de aceptar su papel como "forasteros de la sociedad" (Zeiher et al, 1996). La moratoria psicosocial considerada durante mucho tiempo como un requisito indispensable de la buena infancia, en la que los niños se están preparando para entrar en la vida adulta, está siendo sustituida cada vez más por prácticas en las que los niños ya tienen experiencias similares a las de los adultos y llevan a cabo actividades que antes estaban reservadas solo a los adultos (Liebel, 2006: 47-74). Una infancia que, separada de la vida de los adultos, es entendida sólo como un tiempo de ausencia y de preparación, es hoy entendida por un número creciente de niños como trivial y ha quedado obsoleta. En otras palabras, podría decirse que los antiguos "forasteros" están volviendo a la sociedad y registrando nuevas demandas.

Las sociedades del Norte Global han respondido a estos cambios de manera contradictoria. Por un lado, se reconoce que se han movido "hacia una situación difícil con la aparentemente inevitable exclusión de la infancia de la vida adulta" (Rumpf, 1989: 58). Cada vez más se reconoce que los niños saben y son capaces de hacer más de lo que antes se pensaba que podían hacer, y que las instituciones educativas deben estar 
más estrechamente vinculadas a la vida social y tomar más en serio las experiencias cotidianas de los niños. En las escuelas esto se expresa, por ejemplo, en el apoyo a las "microempresas estudiantiles", en las que los propios niños pueden experimentar el sentido práctico de los conocimientos que deben adquirir. $\mathrm{O}$ en la vida social se subraya la necesidad de la participación de los niños en las decisiones políticas y se invoca su ciudadanía. Pero todos estos procesos se intentan controlar y limitar de tal manera que no pongan en peligro el orden existente. Las microempresas estudiantiles están instrumentalizadas para la práctica del empresariado infantil (Liebel, 2006: 189-208), la propagación de la ciudadanía "activa" está ligada a la ideología neoliberal, según la cual cada uno es el herrero de su propia fortuna (Gaitán y Liebel, 2011). Ni la desigualdad social existente ni la generacional son tocadas o incluso eliminadas. Por el contrario, cuanto más se cuestiona el orden generacional jerárquicamente concebido paternalmente en la "época de incertidumbre" (Lee, 2001) y bajo la influencia de la comunicación globalizada, más esfuerzos se hacen para justificar y consolidar la asimetría entre los viejos y los jóvenes, y el paternalismo adultista como una constante antropológica, supuestamente, parece irreversible (Liebel, 2018).

Se pueden observar contradicciones similares con respecto a las infancias híbridas, promovidas por los medios sociales y los procesos migratorios. Hoy en día, los niños son cada vez más activos a nivel transnacional, a través de los me- dios de comunicación social y forman o participan en redes transnacionales. La difusión de las identidades transnacionales socava la base del proyecto de infancia concebido monoculturalmente por un Estado nacional.

La desigualdad de poder y recursos puede persistir durante algún tiempo en el mundo, pero no justifica el mantenimiento de patrones de vida basados en privilegios materiales, como criterio para una infancia "exitosa". La vieja idea de que la persona adulta ya es "perfecta" -en comparación con la imperfección infantil- ya ha cumplido su propósito, puesto que dicho argumento se usó como la justificación para excluir a aquellos que "aún no estaban preparados" (y crear esta especie de moratoria). Hoy en día, la educación se cuestiona como una mera educación escolar que precede a la supuesta seriedad de la vida y está separada de la vida social. Del mismo modo, el llamado "tiempo libre" ya no puede separarse tan limpiamente de la esfera del trabajo ni entenderse como un refugio de la libertad, como sugiere el término. En lugar de declarar moratorias, por mucho que aún estén arraigadas ideológicamente, ya no pueden seguir siendo un modelo fijo de infancia, se debería pensar más en cómo los intervalos en la vida de los sujetos se pueden organizar de manera igualitaria y equitativa.

\section{Conclusiones}

La investigación sobre la infancia moderna se enfrenta al reto de considerar, de modo lúcido, 
los cambios asociados a la globalización económica y cultural. Tendrá que preguntarse si la idea, inspirada en la teoría de la modernización, de que existen en ciertas sociedades y culturas formas particularmente avanzadas de infancia debe seguir utilizándose como criterio y modelo sostenible para evaluar las diversas infancias alrededor del mundo (si es que alguna vez lo han sido). Por ejemplo, los discursos que recaen sobre determinados "niños sin infancia", producto de que viven en condiciones de vida que difieren de ese modelo europeo, burgués y colonialista de infancia dominante, también estarían escondiendo un sentimiento de la propia superioridad de la sociedad europea, burguesa y colonialista, puesto que no son del todo adecuadas para analizar las diferentes infancias que están surgiendo, sobre todo, en el Sur Global. Si permitimos la idea de que son posibles las infancias que no están externalizadas a esferas de protección y aisladas de la vida de los adultos, será más fácil comprender que tanto los niños como los adolescentes -según cada edad- pueden representar una fuerza que desempeña un papel decisivo en la estructuración y transformación de la vida social.

En vista de las tendencias presentadas, abogo con Cinthya Saavedra y Steven Camicia (2010) por una nueva geopolítica de las infancias que retome los modos de vida, las formas de conocimiento y los potenciales caminos de acción encarnados en las infancias transnacionales ("niños en movimiento"), con sus identidades múltiples e híbridas como un impulso cultural para un mundo abierto, ilimitado, solidario y un orden generacional no paternalista. A pesar de la persistencia de las fronteras nacionales y la compartimentación del Norte Global de los pueblos del Sur Global, más y más personas están en movimiento, mezclándose con personas de otras naciones, culturas e idiomas, y creando nuevas identidades que apenas han existido antes. Los niños y jóvenes que emigran con sus familias o solos a otros países, así como los que viven y regresan temporalmente a ellos, encarnan nuevas experiencias, estilos de vida y formas de pensar que amplían los conocimientos, los horizontes y los recursos económicos y culturales de los países y comunidades donde llegan. Desafían a la geopolítica transnacional al reconocer y promover el papel proactivo de los niños migrantes para la transformación de un mundo segregado y desigual en un mundo sin fronteras ni dominación.

\section{Referencias citadas:}

Balogapalan, S. (2014): Inhabiting 'Childhood': Children, labour and schooling in postcolonial India, Basingstoke y Nueva York, Palgrave Macmillan.

Brand, U. y M. Wissen (2017): Imperiale Lebensweise. Zur Ausbeutung von Mensch und Natur im globalen Kapitalismus, Múnich, Oekom.

Cannella, G. S. y R. Viruru (2004): Childhood and Postcolonization: Power, Education, and Contemporary Practice, Nueva York y Londres, RoutledgeFalmer. 
Comaroff, J. y J. Comaroff (2008): “The Colonization of Consciousness", en M. Lambek, Ed., A Reader in the Anthropology of Religion, Malden, MA, Blackwell, pp. 464-478.

Cregan, K. y D. Cuthbert (2014): Global Childhoods, Los Angeles, Sage.

De Sousa Santos, B. (2009): Una epistemología del Sur: la reinvención del conocimiento y la emancipación social, Buenos Aires, Siglo XXI.

Emond, R. y F. Esser (2015): “Introduction: Transnational childhoods", Transnational Social Review, 5(2), pp. 100-103.

Ennew, J. (2002): "Outside childhood: street children's rights", en B. Franklin, Ed., The New Handbook of Children's Rights, Londres \& Nueva York, Routledge, pp. 388-403.

Gaitán, L. y M. Liebel (2011): Ciudadanía y Derechos de Participación de los Niños, Madrid, Síntesis.

Gstettner, P. (1981): Die Eroberung des Kindes durch die Wissenschaft. Aus der Geschichte der Disziplinierung, Hamburgo, Rowohlt.

Habermas, J. (1981): Teoría de la Acción Comunicativa, Madrid, Taurus.

Hegel, G. W. F. ([1822]1989): Lecciones sobre la filosofía de la historia universal, Madrid, Alianza.

Katz, C. (2004): Growing Up Global: Economic Restruc- turing and Children's Everyday Lives, Minneapolis, University of Minnesota Press.

Katz, C. (2012): "Work and Play: Economic restructuring and children's everyday learning in rural Sudan", en G. Spittler \& M. Bourdillon, Eds., African Children at Work: Working and Learning in Growing Up for Life, Zurich y Berlín, LIT, pp. 227-248.

Kipling, R. (1899): The White Man's Burden. Disponible en web: http://www.loske.org/html/school/history/c19/burden_full.pdf

Lee, N. (2001): Childhood and society: Growing up in an age of uncertainty, Buckingham y Philadelphia, Open University Press.

Lessenich, S. (2017): Neben uns die Sintflut. Die Externalisierungsgesellschaft und ihr Preis, Berlín, Hanser.

Liebel, M. (2006): Malabaristas del siglo XXI. Los niños y niñas trabajadores frente a la globalización, Lima, Ifejant.

Liebel, M. (2018): “Más allá del paternalismo. Hacia una protección participativa y una partici-pación protagónica”, en A. Llena Berñe \& A. M. Novella Cámara, Eds., Impulsar la Participación Infantil. Los consejos de infancia y adolescencia, Barcelona, Editorial GRAÓ, pp. 51-83.

Liebel, M. (2019): Infancias dignas, o cómo descolonizar las infancias, Lima, Ifejant; Buenos Aires, Editorial El Colectivo; México, Bajo Tierra Editores. 
Locke, J. ([1690]1999): Ensayo sobre el entendimiento humano, México, Fondo de Cultura Económica.

Mehta, U. S. (1999): Liberalism and Empire: A Study in Nineteenth-Century British Liberal Thought, Chicago y Londres, University of Chicago Press.

Mendel, G. (1971): Pour décoloniser l'enfant. Sociopsychanalyse de l'autorité, Paris, Payot.

Mendel, G. (1974): La decolonización del niño, Barcelona, Ariel.

Milanović, B. (2012): The Haves and the Have-Nots: A Brief and Idiosyncratic History of Global Inequality, Nueva York, Basic Books.

Osterhammel, J. (2005): Colonialism: A Theoretical Overview, Princeton, NJ, Markus Wiener Publishers.

Pavez-Soto, I. (2013): “Los significados de 'ser niña y niño migrante': conceptualizaciones desde la infancia peruana en Chile", Polis - Revista Latinoamericana, 35. Disponible en web: https://journals.openedition.org/polis/9304 [Consulta: 15 de mayo de 2019].

Piketty, T. (2014): El Capital en el Siglo XXI, Madrid, Fondo de Cultura Económica de España.

PNUD (1999): Informe sobre Desarrollo Humano 1999, Madrid, Mundi-Prensa Libros.

Postman, N. (1988): La desaparición de la niñez, Madrid, Círculo de Lectores.
Pries, L. (2010): Transnationalisierung. Theorie und Empirie grenzüberschreitender Vergesell-schaftung, Wiesbaden, VS Verlag.

Rohr, E. (2013): “La infancia transnacional y el debate en torno a la 'cadena del cuidado'”, Ecuador Debate. Identidades y diferencias, 88, pp. 169-182.

Rousseau, J.-J. ([1762]1998): Emilio, o De la educación, Madrid, Alianza.

Rumpf, H. (1989): "Ernstes Spiel - Mini-München, etwas anderes als eine Kinderbelustigung“, en G. Grüneisl y W. Zacharias, Eds., Die Kinderstadt. Eine Schule des Lebens. Handbuch für Spiel, Kultur, Umwelt, Hamburgo, Rowohlt, pp. 58-65.

Saavedra, C. M. \& S. P. Camicia (2010): “Transnational Childhoods: Bodies that Challenge Boundaries", en G. S. Cannella y L. Diaz Soto, Eds., Childhoods. A Handbook, Nueva York, Peter Lang, pp. 27-37.

Schibotto, G. (2015): "Saber Colonial, Giro Decolonial e Infancias Múltiples de América Latina”, NATs - Revista Internacional desde los Niños, Niñas y Adolescentes Trabajadores, 25, pp. 51-68.

Stearns, P. N. (2005): Growing Up. The History of Childhood in a Global Context, Waco, TX, Baylor University Press.

Stearns, P. N. (2006): Childhood in World History, Nueva York y Londres, Routledge.

UNICEF (2006): Zur Situation der Kinder in der Welt 
2006. Kinder ohne Kindheit, Fráncfort, Fischer.

Walsh, S. (2010): Kipling's Children's Literature: Language, Identity and Constructions of Childhood, Farnham, Ashgate.

Wells, K. (2009): Childhood in a Global Perspective, Cambridge, Malden, MA, Polity Press.

Zeiher, H., P. Büchner y J. Zinnecker, Eds. (1996): Kinder als Außenseiter? Umbrüche in der gesellschaftlichen Wahrnehmung von Kindern und Kindheit, Weinheim y Múnich, Juventa

\section{Notas:}

1Agradezco a Iskra Pavez Soto por la revisión teórica y lingüística y a los dos revisores anónimos por sus comentarios críticos y sugerencias de cambios.

2Locke dice: "(Todas las ideas vienen de la sensación o de la reflexión). Supongamos, entonces, que la mente sea, como se dice, un papel en blanco, limpio de toda inscripción, sin ninguna idea. ¿Cómo llega a tenerlas?".

${ }^{3}$ La doctrina de la "terra nullius" o "tierra de nadie" difundida por primera vez en al año 1096 por el Papa Urbano II en una bula papal para justificar las cruzadas hacia territorios habitados por no-cristianos del mundo conocido en aquella época y autorizar su ocupación, tenía una importancia similar para la legitimación de las conquistas coloniales. Las doctrinas de derecho de los siglos XVI y XVII recurrían al concepto de la "terra nullius" para reconocer los derechos de propiedad sobre territorios que no estaban bajo control de ninguna entidad reconocida por algún poder europeo.

4"The White Man's Burden" o "La carga del hombre blanco " es el título de un poema que Rudyard Kipling publicó a fines del siglo XIX bajo la conquista estadounidense en las Filipinas y de otras ex colonias españolas. Kipling califica a la población indígena de las colonias como "vuestros recién conquistados y descontentos pueblos" y "mitad niños", instando al lector a asumir la carga del "hombre blanco" de liberarlos de su estado salvaje e infantil. El poema es considerado uno de los testimonios más importantes del imperialismo.

${ }^{5}$ No se entienden los términos Sur Global y Norte Global en un sentido geográfico, sino en un sentido geopolítico. En este sentido, Chile, por ejemplo, puede estar ubicado tanto en el Sur como en el Norte Global. 